\title{
Rapid evolution of fluoroquinolone-resistant Escherichia coli in Nigeria is temporally associated with fluoroquinolone use
}

\author{
Adebayo Lamikanra', Jennifer L Crowe ${ }^{2}$, Rebeccah S Lijek², Babatunde W Odetoyin ${ }^{3}$, John Wain ${ }^{4}$, \\ A Oladipo Aboderin ${ }^{3}$ and Iruka N Okeke $2^{2^{*}}$
}

\begin{abstract}
Background: Antibiotic resistance has necessitated fluoroquinolone use but little is known about the selective forces and resistance trajectory in malaria-endemic settings, where selection from the antimalarial chloroquine for fluoroquinolone-resistant bacteria has been proposed.

Methods: Antimicrobial resistance was studied in fecal Escherichia coli isolates in a Nigerian community. Quinolone-resistance determining regions of gyrA and parC were sequenced in nalidixic acid resistant strains and horizontally-transmitted quinolone-resistance genes were sought by PCR. Antimicrobial prescription practices were compared with antimicrobial resistance rates over a period spanning three decades.

Results: Before 2005, quinolone resistance was limited to low-level nalixidic acid resistance in fewer than $4 \%$ of $E$. coli isolates. In 2005, the proportion of isolates demonstrating low-level quinolone resistance due to elevated efflux increased and high-level quinolone resistance and resistance to the fluoroquinolones appeared. Fluoroquinolone resistance was attributable to single nucleotide polymorphisms in quinolone target genes gyrA and/or parC. By 2009, 35 (34.5\%) of isolates were quinolone non-susceptible with nine carrying gyrA and parC SNPs and six bearing identical gnrS1 alleles. The antimalarial chloroquine was heavily used throughout the entire period but E. coli with quinolone-specific resistance mechanisms were only detected in the final half decade, immediately following the introduction of the fluoroquinolone antibacterial ciprofloxacin.

Conclusions: Fluoroquinolones, and not chloroquine, appear to be the selective force for fluoroquinolone-resistant fecal $E$. coli in this setting. Rapid evolution to resistance following fluoroquinolone introduction points the need to implement resistant containment strategies when new antibacterials are introduced into resource-poor settings with high infectious disease burdens.
\end{abstract}

Keywords: antimicrobial resistance, antimicrobial use, quinolone resistance, drug resistance, ciprofloxacin, fluoroquinolones, selective pressure, Nigeria, chloroquine, antimalarial, fluoroquinolone-resistant, Escherichia coli

\section{Background}

Chloroquine has been described as one of the most important drugs ever used to treat an infection. This cheap and effective drug was the primary antimalarial worldwide until resistance emerged in the 1970s [1]. Multiple alleles of a chloroquine resistance transporter gene, $p f c r t$, and a multidrug resistance transporter gene,

\footnotetext{
* Correspondence: iokeke@haverford.edu

2Department of Biology, Haverford College, 370 Lancaster Avenue, Haverford, PA 19041, USA

Full list of author information is available at the end of the article
}

pfmdr1, are associated with chloroquine resistance in Plasmodium falciparum. Resistant $P$. falciparum haplotypes that predominate in sub-Saharan Africa appear to possess a fitness disadvantage, suggesting that they are maintained by selective pressure from chloroquine, which is still heavily used [2,3]. Davidson et al [4] recently proposed that, in addition to the selective pressure for chloroquine-resistant plasmodia, chloroquine's weak antibacterial activity confers sufficient selective advantage for fluoroquinolone resistant Escherichia coli (FQREC), which emerged in rural Guyana in the

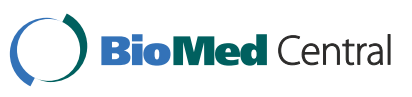


absence of antibacterial quinolone use. If this cheap antimalarial selects for fluoroquinolone-resistant bacteria, justification for reintroduction-by combining it with resistance-reversing chemicals [5] or by withdrawing it until susceptibility is returned and then reintroducing it in combination with other antimalarials [2]-could decrease because of the great and growing need for fluoroquinolones. However, little is known about resistance, drug consumption and selection in malaria-endemic Africa, where high levels of chloroquine and fluoroquinolone use now occurs concurrently.

The quinolone antibacterial nalidixic acid was originally derived from a byproduct of chloroquine synthesis and shares structural features with chloroquine [6]. Nalidixic acid and its more active derivatives, the fluoroquinolones, were rarely used in Africa before the late 1990s because of their high cost. More recently, bacterial resistance to cheaper antibacterials has necessitated widespread fluoroquinolone use. Patents protecting ciprofloxacin and perfloxacin have recently expired, allowing for cheaper generics to be disseminated widely. Fluoroquinolones are stable, orally administrable, and now affordable. In the last decade, fluoroquinolones have become first- and second-line antibacterials of choice for acute respiratory, enteric and urinary tract infections as well as serious systemic infections such as bacteremia $[7,8]$. Fluoroquinolones are also employed in combination with other antimicrobials to treat multidrug resistant tuberculosis $[9,10]$. Additionally, their use and misuse in the informal sector, by unsanctioned providers and through self medication, is commonplace in Nigeria and in many other parts of Africa [11].

Quinolones inhibit the activity of bacterial DNA gyrase and topoisomerase enzymes, which are essential for replication [12]. Single nucleotide polymorphisms in the quinolone resistance determining regions (QRDR) of gyrA and parC, two of the genes that encode DNA gyrase and topoisomerase IV respectively, can lead to conformational changes in these enzymes that prevent quinolones from binding but still preserve their enzymatic function [13]. In E. coli and related Gram-negative bacteria, DNA gyrase is the first target for fluoroquinolones. If gyrA has resistance-conferring mutations, the primary target of fluoroquinolones switches from DNA gyrase to topoisomerase IV $[12,14,15]$. Mutations in the QRDRs of gyrA and parC are the most commonly documented quinolone resistance mechanisms, but resistance is also known to be conferred by mutations in the second topoisomerase gene, parE [14]. Quinolone resistance can also be acquired horizontally through transferable $a a c\left(6^{\prime}\right)-I b$ or quinolone resistance $(q n r)$ DNA. AAC(6')-Ib encodes a ciprofloxacin acetylating enzyme, while the product of $q n r$ inhibits quinolones binding to target proteins $[16,17]$. Another mechanism of quinolone resistance relies on upregulation of efflux pumps that export quinolones and other antimicrobials out of the bacterial cell. For example, mutations in the gene $\operatorname{acr} R$, which encodes a repressor of the $\operatorname{acr} A B$ pump genes, are associated with quinolone resistance [18] Recently, qepA and $o q x A B$, which encode horizontally-transmitted efflux pumps have also been described $[17,19]$.

Low-level resistance to the quinolones often evolves by acquisition of one resistance-conferring mutation or gene. Subsequent genetic changes lead to higher levels of resistance to the first generation quinolone nalidixic acid and a broadening of the resistance spectrum to include second-generation quinolones (first generation fluoroquinolones) such as ciprofloxacin, followed by newer second- and third-generation fluoroquinolones that are yet to reach markets in Nigeria and most other African countries [12,20]. Although multiple mechanisms of quinolone resistance have been reported from other continents, there are very few data from subSaharan Africa on the molecular basis for quinolone resistance. Prior to 2009, horizontally-acquired resistance had not been reported. If cross-selection from chloroquine results in antibacterial resistance more globally, then 1) FQREC should be commonplace in equatorial Africa, which has seen the greatest pressure from chloroquine, and 2) FQREC would have evolved before the introduction of quinolone antibacterials. We report quinolone susceptibility trends among commensal E. coli isolated from apparently healthy undergraduate students over three decades and antimicrobial use at the University health center over that period. Quinolone resistance emerged within the last decade and was coincident with the introduction of the fluoroquinolone ciprofloxacin.

\section{Methods}

\subsection{Bacterial isolates}

Permission to conduct this study was provided by the Institutional Review Board of Obafemi Awolowo University, Ile-Ife, Nigeria. E. coli isolates were recovered from stool specimens collected from apparently healthy undergraduates at Obafemi Awolowo University as described previously [21]. Data from isolates collected in 1986-1998 has been described previously. For this study, we collected and processed specimens in 2005 and 2009 using identical protocols. All volunteers submitting a stool specimen gave informed consent. The identity of colonies with a typical E. coli morphology on MacConkey agar was confirmed by biochemical testing. Colonies from the same specimen with identical biochemical and susceptibility profiles were treated as identical isolates. 


\subsection{Antimicrobial susceptibility testing}

Each strain was tested for susceptibility to eight antimicrobials using the Clinical and Laboratory Standards Institute (CLSI, formerly NCCLS) disc diffusion method [22]. Discs used contained ampicillin $(10 \mu \mathrm{g})$, streptomycin $(10 \mu \mathrm{g})$, trimethoprim $(5 \mu \mathrm{g})$, tetracycline $(30 \mu \mathrm{g})$, nalidixic acid $(30 \mu \mathrm{g})$, chloramphenicol $(30 \mu \mathrm{g})$, ciprofloxacin $(5 \mu \mathrm{g})$ and sulphonamide $(300 \mu \mathrm{g})$ (Oxoid/Remel) and E. coli ATCC 35218 was used as control strain. Inhibition zone diameters were interpreted in accordance with CLSI guidelines using WHONET software version 5.3 [23]. Minimum inhibitory concentrations (MICs) to nalidixic acid were measured using the agar dilution technique on Mueller-Hinton agar as recommended by the CLSI and using E. coli ATCC 35218 as control [24]. MICs to nalidixic acid were also measured in the presence of efflux pump inhibitor phe-arg $\beta$-naphthylamide.

\subsection{Mutational analysis of the quinolone-resistance determining regions of $g y r A$ and parC}

DNA was extracted from each quinolone-resistant isolate, using the Promega Wizard genomic extraction kit. The quinolone-resistance determining regions of the gyr $A$ and parC genes were amplified from DNA templates by PCR using Platinum PCR supermix (Invitrogen) and the primer pairs listed in Additional file 1, Table S1. PCR reactions began with a two-minute hot start at $94^{\circ} \mathrm{C}$ followed by 30 cycles of $94^{\circ} \mathrm{C}$ for $30 \mathrm{~s}$, annealing temperature, $30 \mathrm{~s}$ and $72^{\circ} \mathrm{C}$ for $30 \mathrm{~s}$. gyr $\mathrm{A}$ amplifications were annealed at $58^{\circ} \mathrm{C}$ and parC reactions were annealed at $52^{\circ} \mathrm{C}$. E. coli K-12 MG1655 [25] was used as a control. Amplicons were sequenced on both strands and predicted peptide sequences were compared to the corresponding gene from the MG1655 genome [25] by pair-wise FASTA alignments.

\subsection{Identification of horizontally-acquired quinolone- resistance genes}

$q n r A, q n r B$, and $q n r S$ were identified with PCR using the primer pairs published by Wu et al [26]. The primers of Liu et al [27] were used to screen for the qepA gene (Additional file 1, Table S1). Amplicons were sequence-verified.

\subsection{Organic solvent tolerance}

The qualitative organic solvent tolerance test used by Wang et al [18] was employed. Briefly $10^{3}-10^{4}$ mid-log phase bacteria were spotted onto glass plates of LB agar and overlaid with hexane, cyclohexane or a 1:1 [vol:vol] mixtures of these solvents. Strains were scored as producing no growth under any solvent (-), growth under all solvents $(+++)$ or some solvents $(+$ or ++$)$. Pseudomonas aeruginosa strain PA14 and E. coli MG1655 were used as positive and negative controls respectively.

\subsection{Determination of transcript levels by quantitative real-time PCR}

Total RNA was extracted from late log-phase cultures using the Qiagen RNeasy Protect bacterial minikit. Following reverse transcription using the Taqman Reverse Transcription Reagents and random hexamers (Applied Biosystems), quantitative real-time PCR with SYBR green was performed using primer pairs specific for genes of the components of the efflux pumps or efflux pump regulators using primers of Nishino et al. [28] and Bohnert et al. [29] (Additional file 2, Table S2). The reactions were run on the StepOne Real-time PCR system and performed according to manufacturers recommendations (Applied Biosystems). Gene expression levels were compared to the control strain MG1655, and analyzed using the $2^{-\Delta \Delta C t}$ method [30]. The $\operatorname{rrs} A$ gene was used as the normalizing gene. Each reaction was performed in quadruplicate.

\subsection{Flagellin typing}

fliC PCR-restriction fragment length polymorphism (RFLP) typing was performed as described by Fields et al [31]. The fliC gene of test E. coli strains was amplified using the primers F-FLIC1 and R-FLIC2 (Additional file 1, Table S1). Amplicons were digested with RsaI and restriction profiles were discriminated after electrophoresis on $2.5 \%$ agarose gels.

\subsection{Multi-locus sequence typing}

PCR primers listed in Additional file 1, Table S1 were used to amplify gene fragments from the $a d k$, fum $C$, gyrB, icd, $m d h$, purA and $\operatorname{rec} A$, as described by Wirth et al [32]. Amplified DNA products were sequenced from both ends. Allele assignments were made at the publicly accessible E. coli MLST database accessible from http:// www.mlst.net/. Phylogenetic inferences about ancestral allelic profiles and strain interrelatedness were made using eBURSTv3 at http://eburst.mlst.net/ defining sequence type (ST) complexes, that is clonal complexes, based on groups sharing six identical alleles.

\subsection{Prescription audit of antimicrobial use}

Prescription sheets were obtained with permission from the Obafemi Awolowo University Ile- Ife, Nigeria for 1984, 1985 and 1986; for 1994, 1995 and 1996 and for 2004, 2005 and 2006. Data from the prescription sheets was collated into a spreadsheet anonymously and then the prescription sheets were returned to the University Health Centre. For each year that was evaluated, 300 prescriptions were randomly selected for each month, permitting evaluation of $4-8 \%$ of the prescriptions that were actually filled. Prescriptions for all oral and topical antimicrobials, filled at the institution's Pharmacy were documented and units of measurement were 
standardized to daily-defined doses were computed using the $\mathrm{AB}$ Consumption Calculator tool [33].

\section{Results}

3.1. Emergence of fluoroquinolone resistance among commensal $E$. coli from south-western Nigeria

Our previous studies which tracked resistance in fecal $E$. coli isolates between 1986 and 1998 found uncommon $(0-3.2 \%)$ and, in all but one case, low-level resistance to nalidixic acid and no fluoroquinolone resistance [21]. All but one of the nalidixic acid-resistant isolates identified before 1998 produced an inhibition zone diameter of at least $10 \mathrm{~mm}$ (Nalidixic acid sensitive strains have zone diameters $>18 \mathrm{~mm}$. Resistant strains have zone diameters $<14 \mathrm{~mm}$ ). Due to repeated power cuts in Nigeria, that strain archive is lost and none of those isolates are available for analysis. Employing an identical protocol in 2005 we recovered nalidixic acid non-susceptible E. coli from 18 (21.7\%) volunteers. Four isolates exhibited high-level nalidixic acid resistance, producing no inhibition zone and MICs of $\geq 4 \mu \mathrm{g} \mathrm{ml}^{-1}$, and one of these was fluoroquinolone-resistant (Table 1). The remaining isolates exhibited low-level nalidixic acid resistance similar to that seen with earlier isolates reported previously [21]. No 2005 isolate carried horizontally-transmitted qnr or qepA genes but the four high-level nalidixic acid-resistant strains had mutations in the quinolone-resistance determining regions (QRDR) of quinolone target gyrA and the single FQREC isolate had an S80I substitution in ParC (Table 1).

In 2009, we recovered nalidixic acid-resistant E. coli from 35 (34.5\%) volunteers of which 11 isolates were fluoroquinolone non-susceptible. Isolates obtained from nine individuals in 2009 harbored one or more nonsynonymous mutations in $g y r A$ and parC QRDRs and six isolates from the same year carried the horizontally- transmitted quinolone-resistance gene qnrS1 [34] (Table 1). Altogether, quinolone-specific mechanisms of resistance were identified in 16 isolates from 15 (17.4\%) individuals in 2009, compared to $4(4.8 \%)$ in 2005 (p < 0.05, Chitest with Yates correction for small numbers). All FQREC strains carried two or more QRDR mutations and/or qnrS1 (Table 1).

\subsection{Molecular basis for nalidixic acid resistance in isolates obtained in 2005, during the transition to \\ fluoroquinolone resistance}

We isolated 21 nalidixic-acid resistant $E$. coli strains from 18 volunteers in Nigeria in 2005 and one of these was resistant to the fluoroquinolone ciprofloxacin. The majority (17) showed low-level nalidixic acid resistance and did not have mutations in $\operatorname{gyr} A$ or $\operatorname{parC}$, nor did they carry horizontally-transmitted quinolone resistance $q n r$ and qepA genes. Most of these isolates showed undue tolerance to organic solvents, suggesting that overactive efflux pumps may account for resistance (Table 2). We measured nalidixic acid MICs by agar dilution in the absence and presence of the RND-type efflux pump inhibitor phe-arg $\beta$-naphthylamide. As shown in Table 2 test strains showed an 8 -fold or greater reduction in the concentration of nalidixic acid needed to inhibit growth in the presence of phe-arg $\beta$ naphthylamide, strongly suggesting a role for up-regulated efflux pumps. To confirm efflux pump upregulation, we used quantitative real-time PCR (qPCR) to measure transcription of 13 genes representing the major known efflux systems in $E$. coli. Compared to $E$. coli $\mathrm{K}-12$ strain MG1655, sixteen isolates showed a twofold or greater upregulation in transcription of the outer-membrane extrusion factor gene tolC. Seventeen of the isolates showed two-fold or greater increases in transcription of one or more other efflux genes and at

Table 1 Quinolone-specific resistance mechanisms in 2005 and 2009 nalidixic acid-resistant isolates

\begin{tabular}{|c|c|c|c|c|c|}
\hline Number of isolates & Nalidixic acid MIC & Ciprofloxcin & GyrA SNPs & ParC SNPs & Horizontally-acquired \\
\hline \multicolumn{6}{|l|}{2005} \\
\hline 3 & $8-32$ & Sensitive & S83L & - & - \\
\hline 1 & 1024 & Resistant & S83L, D87N & S801 & - \\
\hline \multicolumn{6}{|l|}{2009} \\
\hline 2 & 256 & Sensitive & D101G* & - & - \\
\hline 1 & 16 & Sensitive & S83L & - & - \\
\hline 1 & 1024 & Resistant & S83L, D87N & - & - \\
\hline 4 & $>1024$ & Resistant & S83L, D87N & $\mathrm{S} 80 \mathrm{l}$ & - \\
\hline 1 & 64 & Sensitive & - & S94F* & - \\
\hline 1 & 256 & Sensitive & - & V54 $1^{*}$ & - \\
\hline 1 & 32 & Resistant & - & $Y 74 C^{*}$ & anrs1 \\
\hline 5 & $16-256$ & Resistant & - & - & anrst \\
\hline
\end{tabular}

*Previously unreported SNP for which there is no evidence of functional contribution to resistance 
Table 2 Multilocus sequence types and antimicrobial efflux mechanisms in 2005 quinolone-resistant isolates

\begin{tabular}{|c|c|c|c|c|c|c|c|c|c|c|c|c|c|}
\hline $\begin{array}{l}\text { QREC } \\
\text { Strain }\end{array}$ & $\begin{array}{l}\text { MLST } \\
\text { ST }\end{array}$ & $\begin{array}{c}\text { ST } \\
\text { Complex }\end{array}$ & $\begin{array}{l}\text { flic } \\
\text { type }\end{array}$ & $\begin{array}{l}\text { Resistance } \\
\text { Profile }\end{array}$ & $\begin{array}{c}\text { Nalidixic } \\
\text { acid MIC } \\
(\mu \mathrm{g} / \mathrm{ml})\end{array}$ & $\begin{array}{l}\text { Organic } \\
\text { solvent } \\
\text { tolerance }\end{array}$ & $\begin{array}{c}\text { Nalidixic acid } \\
\text { fold inhibition } \\
\text { with PABN }\end{array}$ & $\stackrel{\Delta}{\Delta y r A}$ & $\begin{array}{c}\Delta \\
\text { ParC }\end{array}$ & $\begin{array}{l}\text { Transcription } \\
\text { fold change- } \\
\text { tolC }\end{array}$ & $\begin{array}{c}\text { Efflux pump genes } \\
\text { with transcription fold } \\
\text { change } \geq 4\end{array}$ & $\begin{array}{l}\text { Efflux regulator } \\
\text { genes with } \\
\text { transcription fold } \\
\text { change } 2-8\end{array}$ & $\begin{array}{l}\text { Efflux regulator } \\
\text { genes with } \\
\text { transcription fold } \\
\text { change }>8\end{array}$ \\
\hline $01 \mathrm{~A}$ & 10 & 10 & A & A NSLTR & 32 & + & 4 & S83L & - & 2.0 & emrk & - & $r o b$ \\
\hline $05 \mathrm{C}$ & 10 & 10 & $B$ & NS TR & 4 & ++ & 8 & - & - & 1.0 & $m d t A$ & - & - \\
\hline $23 \mathrm{~A}$ & 10 & 10 & $B$ & AC NSLTR & 4 & ++ & 8 & - & - & 2.0 & $m d t A$ & $\begin{array}{c}\text { marA, soxs, beas, } \\
\text { mark, acrR }\end{array}$ & - \\
\hline $33 \mathrm{~A}$ & 10 & 10 & B & AC NSLTR & 4 & ++ & 8 & - & - & 4.5 & $\begin{array}{c}\text { acrA, acrD, acrE, bcr, } \\
\text { cusB, emrA, emrD, emrk, } \\
\text { fsr, }\end{array}$ & $\begin{array}{c}\text { marA, soxs, evg } A, \\
\text { marR, acrR }\end{array}$ & rob, baes \\
\hline $25 \mathrm{~A}$ & 10 & 10 & D & A NSLTR & 4 & ++ & 8 & - & - & 16.8 & $\begin{array}{l}\text { acrA, acrD, acrE, bcr, } \\
\text { cusB, emrA,emrD, emrk, } \\
f s r, m a c A, m d f A, m d t A\end{array}$ & $\operatorname{evg} A$ & $\begin{array}{c}\text { marA, soxs, rob, beas, } \\
\text { marR, acrR }\end{array}$ \\
\hline $40 B$ & 10 & 10 & $E$ & $\mathrm{~N}$ & 4 & +++ & 8 & - & - & 1.0 & - & - & - \\
\hline $16 \mathrm{~A}$ & 34 & 10 & $E$ & NS & 4 & +++ & 16 & - & - & 4.2 & $\begin{array}{l}\text { acrA, acrD, acrE, bcr, } \\
\text { cusB, fsr, mdtA }\end{array}$ & $\begin{array}{c}\text { marA, soxs, rob, marR, } \\
\text { acrR }\end{array}$ & baes \\
\hline $49 \mathrm{~A}$ & 43 & 10 & $\mathrm{~F}$ & $\mathrm{~N}$ & 4 & + & 8 & S83L & - & 2.2 & - & - & - \\
\hline $23 \mathrm{~B}$ & 10 & 10 & $C$ & C N LTR & 4 & & 8 & - & - & 2.2 & - & - & - \\
\hline $31 \mathrm{~A}$ & 494 & none & C & A NSLTR & 4 & ++ & 8 & - & - & 2.2 & $\begin{array}{c}a c r D, a c r E, b c r, e m r A, \\
m d t A\end{array}$ & baes & - \\
\hline $38 \mathrm{C}$ & 521 & none & C & NS R & 4 & ++ & 8 & - & - & 4.3 & $\begin{array}{c}\text { acrA, acrD, acrE, bcr, } \\
\text { cusB, emrA, emrD, fsr, } \\
\text { mdfA, } m d t A\end{array}$ & $\begin{array}{c}\text { marA, soxs, evgA, rob, } \\
\text { marR, acrR }\end{array}$ & beas, \\
\hline $22 \mathrm{~A}$ & 452 & none & C & NSLTR & 4 & + & 8 & - & - & 1.1 & - & - & - \\
\hline $31 \mathrm{~B}$ & 452 & none & C & NSLTR & 4 & - & 4 & - & - & 2.5 & - & - & - \\
\hline $33 B$ & 504 & none & K & NSLT & 1024 & +++ & 16 & S83L & - & 1.0 & - & - & - \\
\hline $34 \mathrm{~B}$ & 498 & 467 & G & NS & 4 & ++ & 8 & - & - & 2.1 & - & baes & - \\
\hline $32 \mathrm{C}$ & 503 & 13 & $\mathrm{H}$ & NSLTR & 4 & ++ & 8 & - & - & 1.0 & - & baes & - \\
\hline $30 \mathrm{~A}$ & 156 & 156 & 1 & AC NSLTR & 4 & ++ & 16 & - & - & 4.3 & $\begin{array}{c}a c r A, a c r D, c u s B, e m r A, \\
\text { emrk, mdfA }\end{array}$ & soxs, baes & - \\
\hline $27 \mathrm{~A}$ & 448 & 448 & J & ACPNSLTR & 1024 & +++ & 2 & $\begin{array}{l}\text { S83L, } \\
\text { D87N }\end{array}$ & S801 & 33.2 & $\begin{array}{l}\text { acrA, acrD, acrE, bcr, } \\
\text { cusB, emrA, emrD, fsr, } \\
\text { macA, mdfA, mdtA }\end{array}$ & - & $\begin{array}{c}\text { marA, soxs, evgA, rob, } \\
\text { beas, marR, acrR }\end{array}$ \\
\hline $09 \mathrm{C}$ & 517 & none & L & AC NSLTR & 4 & ++ & 8 & - & - & 8.6 & $\begin{array}{l}\text { acrA, acrD, acrE, bcr, } \\
\text { cusB, emrA,emrD, emrk, } \\
\text { fsr, mdfA, mdtA }\end{array}$ & $\begin{array}{c}\text { marA, soxs, evgA, rob, } \\
\text { marR, acrR }\end{array}$ & beas \\
\hline $38 \mathrm{~A}$ & 517 & none & N & A NSLTR & 4 & ++ & 8 & - & - & 4.2 & $\begin{array}{c}a c r R, a c r D, \text { acre, bcr, } \\
\text { cusB, fsr, mdtA }\end{array}$ & $\begin{array}{c}\text { marA, soxS, evgA, rob, } \\
\text { baes, marR, acrR }\end{array}$ & \\
\hline $\begin{array}{l}\text { ATCC } \\
25922\end{array}$ & ND & ND & ND & - & 2 & - & 8 & ND & ND & 0.4 & - & - & - \\
\hline CTC10418 & ND & ND & ND & - & 2 & - & 4 & ND & ND & 2.1 & - & - & - \\
\hline
\end{tabular}


Table 3 Prescribing patterns of physicians in the University Health Center

\begin{tabular}{lccc}
\hline & $\begin{array}{c}\mathbf{1 9 8 4 -} \\
\mathbf{1 9 8 6}\end{array}$ & $\begin{array}{c}\mathbf{1 9 9 4 -} \\
\mathbf{1 9 9 6}\end{array}$ & 2004-2006 \\
\hline $\begin{array}{l}\text { Number of prescribing } \\
\text { physicians }\end{array}$ & 7 & 10 & 9 \\
\hline $\begin{array}{l}\text { Mean } \pm \text { SD drugs per } \\
\text { prescription }\end{array}$ & $3.1 \pm 1.1$ & $3.2 \pm 0.5$ & $4.5 \pm 0.5$ \\
\hline $\begin{array}{l}\text { Median (Range for different } \\
\text { physicians) drugs per } \\
\text { prescription }\end{array}$ & $3.1(1.6-5)$ & $3.25(2.5-4)$ & $4.71(3.5-4.9)$ \\
\hline
\end{tabular}

least half of the targets were upregulated in most isolates (Table 2). The acrA efflux pump gene and $a c r R$, encoding the gene's transcriptional regulator, and their promoters, were sequenced from five strains but none contained nucleotide polymorphisms that could account for increased efflux. We next examined transcription of six genes encoding regulators of efflux genes, including acrR, by qPCR and most strains showed upregulated transcription of one or more of these genes (Table 2). Of the three E. coli strains we employed as controls, strains ATCC 25922 and MC4100 produced fold changes for all genes ranging between 0.5 and 1.4 (compared to K-12 strain MG1655) for the different genes but we saw a two-fold increase for NCTC 10418 for nine efflux pump genes but none of the efflux regulators. Twelve of the QREC from Nigeria showed a fourfold or greater fold change in transcription of at least one gene encoding an efflux pump component and all but one of these strains had a two-fold of greater fold upregulation of one or more regulators. Thus, elevated efflux is the most likely explanation for low-level resistance seen in most of the 2005 isolates.

MLST of quinolone-resistant E. coli revealed a startling lack of diversity with the 21 strains representing only 13 sequence types. Nine isolates belonging to sequence type (ST) complex 10 and seven specifically belonging to ST 10. ST452 (2 isolates) and its single locus variant ST521, represented a second frequently occurring clonal complex and we identified one ST156 strain, which along with ST10 has recently been identified among quinolone-resistant $E$. coli in Ghana (Table 2) [35].

\subsection{Antimicrobial prescription trends at the University Health center}

As shown in Table 3, prescribing practices at the Health Center were highly physician-specific in the 1980s but by 2004-2006, had become more uniform. However, with the increasing uniformity, we observed an overall increase in the mean and median number of drugs per prescription (Table 3). While only one of five physicians averaged more than four drugs per prescription in 19841986, in 2004-2006, seven of nine physicians were prescribing an average of four drugs or more per patient. In spite of the very small number of prescribing physicians, this difference approached statistical significance ( $p=0.06$ Fishers exact test). Anti-infectives, the most commonly prescribed medicines at the health center, represented $49.3 \%$ of the drugs listed on prescriptions between 1984 and $1986,21.9 \%$ in $1994-1996$ and $38.7 \%$ in 2004-2006.

In the 1980s, chloroquine accounted for over $96 \%$ of all antimalarials prescribed. Pyrimethamine alone was prescribed in prophylactic doses only and sulphadoxinepyrimethamine, which is more expensive than chloroquine but much cheaper than other antimalarials, was rarely prescribed during the first decade. In the second decade of the study, this drug combination was prescribed for $5 \%$ of patients receiving an antimalarial and by the 2000s, it was recommended for almost $40 \%$ (Figure 1). With the exception of Artemisinin Combination Therapies (ACTs), use of which increased considerably between 2004 and 2006, there were no significant within-decade differences in prescription rates for each antimalarial. However, antimalarial prescription choices changed considerably from one decade to the next. Discounting combinations, the greatest diversity of antimalarial drugs was prescribed in the middle decade, 1990s (Figure 1) and may have been prompted by emerging resistance and the absence of a set antimalarial policy. Amodiaquine and halofantrine represented a significant proportion of prescriptions in the 1990s, but by the 2000s, all patients not receiving chloroquine or amodiaquine received ACTs [36]. A decline in chloroquine prescription from $45.4 \%$ to $25.1 \%$ occurred in parallel with ACT roll out. However chloroquine prescription was never discontinued and it remained the antimalarial of choice for most patients with clinical malaria throughout the duration of the study (Figure 1).

Like antimalarial prescription, antibacterial prescription altered significantly over the study period (Figure 1). Chloramphenicol and tetracycline, drugs of choice in the 1980s, were prescribed less in the following decades as drugs that did not have toxicity concerns became available and more affordable. Prescription of the aminopenicillins ampicillin and amoxicillin for example increased eight-fold from between the first and last decade. As with antimalarials, with the exception of tetracycline, drugs that fell out of favor were never completely discontinued so that selective pressure from them was never removed. Ciprofloxacin was the only quionolone prescribed at the health center and was introduced in the 2000s, the decade in which E. coli with quinolonespecific resistance mechanisms were detected. 


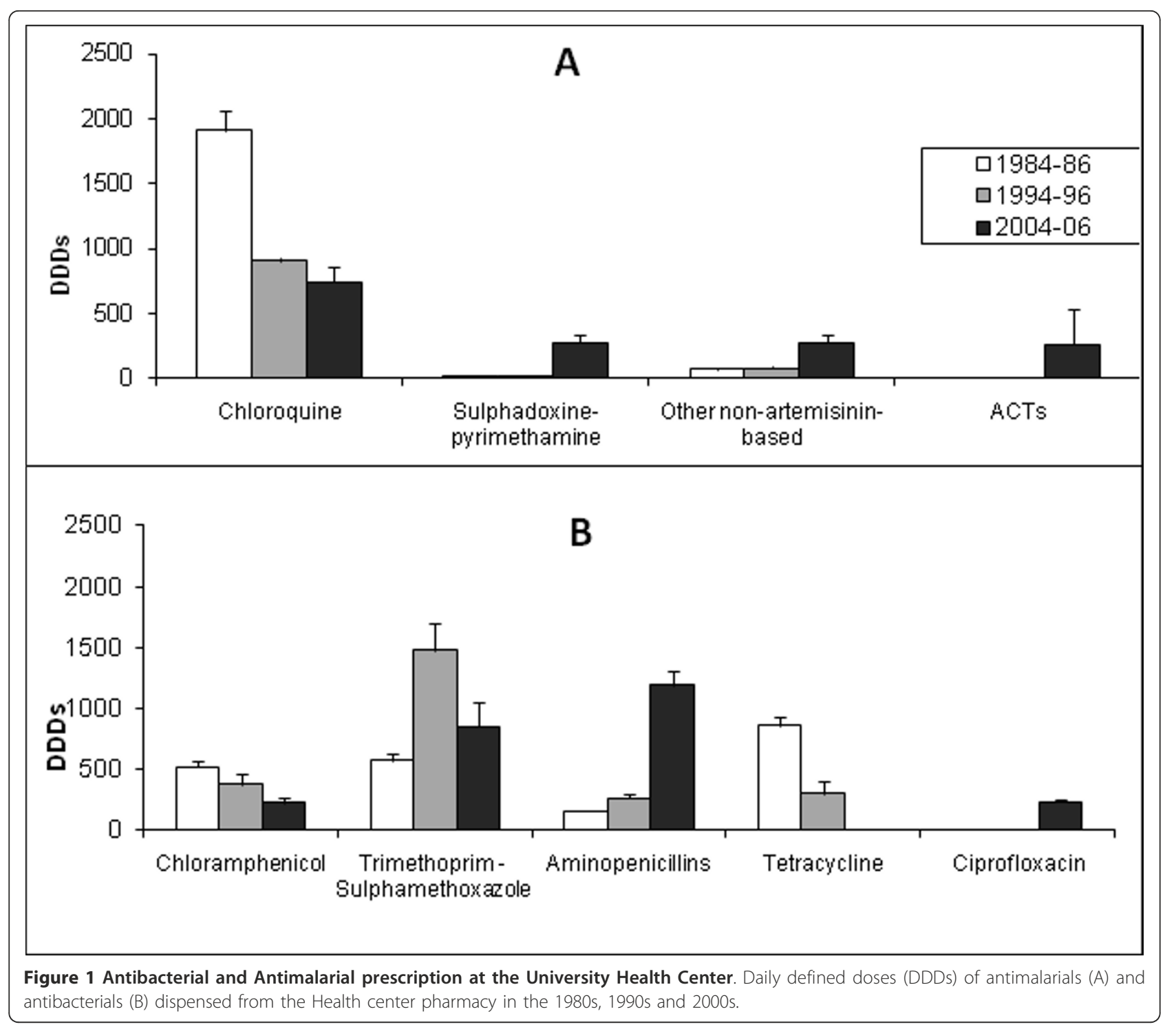

\section{Discussion}

This study allowed us to broach the almost untestable question of population-level selective forces for quinolone resistance in bacteria within a malaria-endemic area. The long duration of resistance monitoring offered significant advantages in spite of unavoidable limitations. We deemed it important to replicate the methodology of earlier years, which means that we only examined a small sample of the commensal E. coli population carried by each volunteer. Other limitations of this study include the fact that our prescription audit did not sample the complete catalogue of selective pressure for resistance. It is unlikely that patients who receive free medications at the health center would have chosen to purchase their prescriptions elsewhere, however, we cannot rule out the possibility that patients may have purchased other medicines to augment, or replace, those offered at the health center. Prescriptions for parenteral drugs are handled differently and records are not retained long-term at the center and therefore injectables were not evaluated, even though it is well known that many commonly used antimicrobials are administered intramuscularly in Nigeria [37].

The quinolone resistance rates and their association with access to quinolones that we have recorded in this study compare with those from reports from other parts of Nigeria and West Africa, and all support the hypothesis that fluoroquinolone introduction immediately precedes the evolution of FQREC [38-42]. However, it is difficult to draw even cautious conclusions across multiple studies and or from anecdotes. Therefore, in spite of the aforementioned limitations, the present study provides coherent evidence to support the hypothesis because it tracks resistance and use in a select area 
using simple but reproducible methodology. Our data show that quinolone resistance emerged in commensal E. coli after the 1990s in Ile-Ife, Nigeria, and that our 2005 isolate collection, largely but not entirely comprised of low-level resistors, appears to have been procured at a critical juncture-shortly after the evolution of resistance. By 2009, 16 (46\%) of quinolone-resistant isolates showed high-level quinolone resistance, and resistance to the fluoroquinolones, attributable to quinolonespecific resistance mechanisms that have commonly been reported in other studies. Resistance-conferring mutations in gyrA and parC were the most commonly recorded of these resistance mechanisms. When horizontally-transmitted resistance evolved, qnrS1 was the most frequently detected transmissible allele among commensal E. coli from Vietnam and Ghana, where like Nigeria antimicrobials are freely available and fecal-oral transmission of bacteria is common $[35,43]$. Altogether, we observed rapid, stepwise evolution of FQREC between 2005 and 2009, from a very low baseline.

The predominance of ST10 among 2005 quinolone resistant isolates may be due to the overall predominance of ECOR phylogenetic group A strains in West Africa [44]. However, we have recently shown that this lineage is overrepresented among quinolone-resistant strains, as compared to sensitive strains in Ghana [35]. We performed in-depth analyses of the 2005 isolates from this study to get a sense of how resistance might have evolved and our data suggest that strains with overactive efflux may have been the predecessors of strains exhibiting quinolone-specific resistance mechanisms. The differences in efflux phenotypes, efflux genes transcription profiles and flagellin types among most of the strains belonging to the same ST suggest that the low-level resistance we detect is not primarily due to expansion of recently derived clones. Instead, the data appear to suggest that elevated efflux is more likely to be seen in specific lineages, likely those with overactive efflux pumps. This in turn points to the idea that certain lineages may be primed to evolve more stable, less costly resistance. Horizontally-transmitted $q n r$ genes, including qurS1 identified in this study have recently been implicated in providing low-level quinolone resistance that could provide strains with the opportunity to acquire subsequent mutations in quinolone targets and therefore higher-level stable resistance [45]. It is therefore worrisome that qurS1 can now be commonly detected among E. coli commensals in Nigeria. More studies are needed to improve our understanding of the evolution and dissemination of quinolone resistance. We suggest that on-going and proposed studies tracking resistance across Africa include a focus on quinolone resistance at genetic as well as phenotypic levels and that more work is done to elucidate the genetic context of successful horizontally transmitted quinolone resistance genes as they appear in African countries, similar to work that has been done in Vietnam [43].

Rampant non-prescription use, informal supply chains and a dearth of archival records make it challenging to quantify drug use in Nigeria. However, the health center at the University where we tracked resistance offers students and staff free health care and archives dispensed prescriptions. This long-term study was thus conducted in a setting that provided the rare opportunity to retrospectively study antimicrobial prescription patterns in an equatorial Africa locality where resistance was also tracked. We find that chloroquine prescription was inordinately high in the 1980 s with $97 \%$ of patients visiting the health center receiving this drug. Chloroquine prescription declined steadily thereafter but never dropped below 500 daily-defined doses, with $25 \%$ of patients receiving this drug in 2006 (Figure 2). In contrast to the earlier report from Guyana [4], we do not find evidence of selection for FQREC from heavy and long-running chloroquine use in this setting. By contrast, quinolone antibacterials were never used before the 2000s and introduction of ciprofloxacin is temporally associated with evolution, and rising rates, of FQREC carriage by campus undergraduates. The significance of our findings cannot be over-emphasized since current efforts to 'bring back' chloroquine, an extremely effective and cheap antimalalarial, would be de-prioritized if there were adverse consequences on resistance in other organisms.

The number of drugs prescribed per patient and the common use of antimicrobial drugs we documented compare with studies performed elsewhere in Nigeria for the respective periods $[46,47]$. Overall antimicrobial

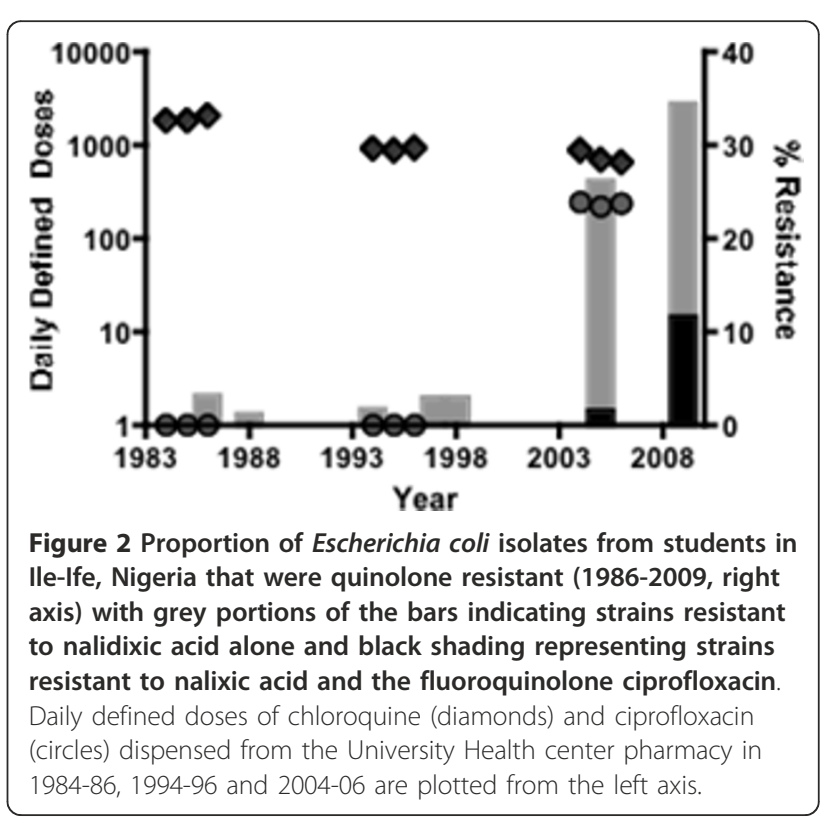


use was characterized by heavy dependence on three drug classes or less and a shift in favored drug categories from one decade to another, without withdrawal of earlier favorites. This drug use pattern, typified by the antimalarial chloroquine, favors the emergence of stable resistance $[40,48,49]$.

Quinolones are drugs of last resort that are increasingly employed in Nigeria and other African countries to manage common bacterial infections. In the face of high and rising resistance to more affordable antimicrobials and the unavailability of newer, patent protected antibacterials, there is currently no real alternative to their use in West Africa. Evolution of resistance to the quinolones among $E$. coli has important clinical implications because resistance patterns in commensals typically mirror those seen in pathogens exposed to similar selection. A recent report from neighboring Cameroon suggests that quinolone resistance has emerged among Salmonella enterica serovar Typhi there [50] and we have reported quinolone resistance in epidemic Vibrio cholerae from Ghana [51].

\section{Conclusion}

This study charts the appearance of quinolone-resistant E. coli in a Nigerian malaria endemic community, which has seen heavy and long-term use of chloroquine. The study demonstrates that quinolone resistance was not temporally associated with chlrooquine use but appeared shortly after fluoroquinolone introduction. The diversity of quinolone-specific resistance mechanisms that emerged following ciprofloxacin introduction points to an urgent need for therapeutic alternatives and resistance containment. Our findings suggest that the useful lifespan for newly introduced antimicrobials that have been used extensively elsewhere can be short. High prices of patent-protected drugs delay their introduction to developing countries and strategies for ensuring early but controlled access to novel antimicrobials may be necessary to ensure medium- to long-term efficacy in resource-limited settings.

\section{Additional material}

Additional file 1: Table S1: Oligonucleotide primers used for standard $P C R$ reactions.

Additional file 2: Table S2: qPCR primers.
This work was supported by International Program In the Chemical Sciences grant NIG01 to AL and by a Branco Weiss Fellowship to INO. JW was supported by the Wellcome Trust and RSL by undergraduate researcher support to Haverford College from the Howard Hughes Medical Institute and the Arnold and Mabel Beckman Foundation.

\section{Author details}

${ }^{1}$ Faculty of Pharmacy, Obafemi Awolowo University, Ile-lfe, Osun State, Nigeria. ${ }^{2}$ Department of Biology, Haverford College, 370 Lancaster Avenue, Haverford, PA 19041, USA. ${ }^{3}$ Department of Medical Microbiology and Parasitology, Obafemi Awolowo University, Ile-Ife, Osun State Nigeria. ${ }^{4}$ Health Protection Agency, Colindale, London, NW9 5EQ, UK.

\section{Authors' contributions}

$\mathrm{AL}$ co-conceived the study, collected isolates and collected antimicrobial use data. JLC designed and performed molecular experiments, analysed and interpreted data. RSL performed molecular experiments, analysed and interpreted data, and contributed to writing the paper. BWO and AOA collected isolates and performed microbiology experiments. JW designed experiments and contributed to writing the paper. INO co-conceived the study, performed microbiology and molecular experiments, analysed and interpreted data and wrote the manuscript. All authors read and approved the final manuscript.

\section{Competing interests}

INO is an editor of BMC Infectious Diseases. No other competing interests are declared by all authors.

Received: 25 June 2011 Accepted: 7 November 2011

Published: 7 November 2011

\section{References}

1. Wellems TE, Plowe CV: Chloroquine-resistant malaria. J Infect Dis 2001, 184(6):770-776.

2. Kublin JG, Cortese JF, Njunju EM, Mukadam RA, Wirima JJ, Kazembe PN, Djimde AA, Kouriba B, Taylor TE, Plowe CV: Reemergence of chloroquinesensitive Plasmodium falciparum malaria after cessation of chloroquine use in Malawi. J Infect Dis 2003, 187(12):1870-1875.

3. Sá JM, Twu O, Hayton K, Reyes S, Fay MP, Ringwald P, Wellems TE: Geographic patterns of Plasmodium falciparum drug resistance distinguished by differential responses to amodiaquine and chloroquine. Proc Nat AcadSci 2009, 106(45):18883-18889.

4. Davidson RJ, Davis I, Willey BM, Rizg K, Bolotin S, Porter V, Polsky J, Daneman N, McGeer A, Yang P, et al: Antimalarial therapy selection for quinolone resistance among Escherichia coli in the absence of quinolone exposure, in tropical South America. PLOS ONE 2008, 3(7):e2727.

5. Henry M, Alibert S, Orlandi-Pradines E, Bogreau H, Fusai T, Rogier C, Barbe J, Pradines B: Chloroquine resistance reversal agents as promising antimalarial drugs. Curr Drug Targets 2006, 7(8):935-948.

6. Lesher GY, Froelich EJ, Gruett MD, Bailey JH, Brundage RP: 1,8Naphthyridine derivatives. A new class of chemotherapeutic agents. J Med Pharm Chem 1962, 91:1063-1065

7. Thielman NM, Guerrant RL: Clinical practice. Acute infectious diarrhea. $N$ Engl J Med 2004, 350(1):38-47.

8. Jamison DT, World Bank, Disease Control Priorities Project: Disease control priorities in developing countries. 2 edition. New York Washington, DC: Oxford University Press; World Bank; 2006.

9. Monedero I, Caminero JA: MDR-/XDR-TB management: what it was, current standards and what is ahead. Expert Rev Respir Med 2009, 3(2):133-145

10. von Gottberg A, Klugman KP, Cohen C, Wolter N, de Gouveia L, du Plessis M, Mpembe R, Quan V, Whitelaw A, Hoffmann R, et al: Emergence of levofloxacin-non-susceptible Streptococcus pneumoniae and treatment for multidrug-resistant tuberculosis in children in South Africa: a cohort observational surveillance study. The Lancet 2008, 371(9618):1108-1113.

11. van den Boogaard J, Semvua HH, Boeree MJ, Aarnoutse RE, Kibiki GS: Sale of fluoroquinolones in northern Tanzania: a potential threat for fluoroquinolone use in tuberculosis treatment. J Antimicrob Chemother 2010, 65(1):145-147.

12. Hooper DC, Rubinstein E: Quinolone antimicrobial agents. 3 edition. Washington, D.C.: ASM Press; 2003. 
13. Hawkey PM: Mechanisms of quinolone action and microbial response. J Antimicrob Chemother 2003, 51(Suppl 1):29-35.

14. Hopkins KL, Davies RH, Threlfall EJ: Mechanisms of quinolone resistance in Escherichia coli and Salmonella: recent developments. Int J Antimicrob Agents 2005, 25(5):358-373.

15. Hooper DC: Mechanisms of action of antimicrobials: focus on fluoroquinolones. Clin Infect Dis 2001, 32(Suppl 1):S9-S15.

16. Tran JH, Jacoby GA: Mechanism of plasmid-mediated quinolone resistance. Proc Natl Acad Sci USA 2002, 99(8):5638-5642.

17. Strahilevitz J, Jacoby GA, Hooper DC, Robicsek A: Plasmid-mediated quinolone resistance: a multifaceted threat. Clin Microbiol Rev 2009, 22(4):664-689.

18. Wang H, Dzink-Fox JL, Chen M, Levy SB: Genetic characterization of highly fluoroquinolone-resistant clinical Escherichia coli strains from China: role of acrR mutations. Antimicrob Agents Chemother 2001, 45(5):1515-1521.

19. Yamane K, Wachino J-i, Suzuki S, Kimura K, Shibata N, Kato H, Shibayama K, Konda T, Arakawa Y: New plasmid-mediated fluoroquinolone efflux Pump, QepA, found in an Escherichia coli clinical isolate. Antimicrob Agents Chemother 2007, 51(9):3354-3360.

20. Morgan-Linnell SK, Zechiedrich L: Contributions of the combined effects of topoisomerase mutations toward fluoroquinolone resistance in Escherichia coli. Antimicrob Agents Chemother 2007, 51(11):4205-4208.

21. Okeke I, Fayinka S, Lamikanra A: Antibiotic resistance trends in Escherichia coli from apparently healthy Nigerian students (1986-1998). Emerg Infect Dis 2000, 6(4):393-396.

22. NCCLS: Performance standards for antimicrobial disk susceptibility tests, 8th Edition; Approved standard. Villanova PA: National Committee for Clinical Laboratory Standards; 2003, 130.

23. O'Brien TF, Stelling JM: WHONET: an information system for monitoring antimicrobial resistance. Emerg Infect Dis 1995, 1(2):66.

24. CLSI: Methods for dilution antimicrobial susceptiblity tests for bacteria that grow aerobically. Approved standard. 7 edition. Wayne, PA: Clinical and Laboratory Standards Institute; 2006.

25. Blattner FR, Plunkett G, Bloch CA, Perna NT, Burland V, Riley M, ColladoVides J, Glasner JD, Rode CK, Mayhew GF, et al: The complete genome sequence of Escherichia coli K-12. Science 1997, 277(5331):1453-1474

26. Wu J-J, Ko W-C, Tsai S-H, Yan J-J: Prevalence of plasmid-mediated quinolone resistance determinants QnrA, QnrB, and QnrS among clinical isolates of Enterobacter cloacae in a Taiwanese hospital. Antimicrob Agents Chemother 2007, 51(4):1223-1227.

27. Liu J-H, Deng Y-T, Zeng Z-L, Gao J-H, Chen L, Arakawa Y, Chen Z-L: Coprevalence of plasmid-mediated quinolone resistance determinants QepA, Qnr, and AAC(6 $\left.6^{\prime}\right)-\mathrm{lb}$-cr among $16 \mathrm{~S}$ rRNA methylase RmtBproducing Escherichia coli isolates from pigs. Antimicrob Agents Chemother 2008, 52(8):2992-2993.

28. Nishino $K$, Senda Y, Yamaguchi A: CRP regulator modulates multidrug resistance of Escherichia coli by repressing the $m d t E F$ multidrug efflux genes. J Antibiot (Tokyo) 2008, 61(3):120-127.

29. Bohnert JA, Schuster S, Seeger MA, Fahnrich E, Pos KM, Kern WV: Sitedirected mutagenesis reveals putative substrate binding residues in the Escherichia coli RND efflux pump AcrB. J Bacteriol 2008, 190(24):8225-8229.

30. Livak KJ, Schmittgen TD: Analysis of relative gene expression data using real-time quantitative PCR and the 2(-Delta Delta $C(T))$ Method. Methods 2001, 25(4):402-408.

31. Fields P, Blom K, Hughes $H$, Helsel L, Feng P, Swaminathan B: Molecular characterization of the gene encoding $\mathrm{H}$ antigen in Escherichia coli and development of a PCR-restriction fragment length polymorphism test for identification of E. coli 0157:H7 and O157:NM. J Clin Microbiol 1997, 35:1066-1070.

32. Wirth T, Falush D, Lan R, Colles F, Mensa P, Wieler LH, Karch H, Reeves PR, Maiden MC, Ochman H, et al: Sex and virulence in Escherichia coli: an evolutionary perspective. Mol Microbiol 2006, 60(5):1136-1151.

33. Monnet D: ABC Calc-Antibiotic consumption calculator. Copenhagen: Statens Serum Institute; 3.02005.

34. Hata M, Suzuki M, Matsumoto M, Takahashi M, Sato K, Ibe S, Sakae K. Cloning of a novel gene for quinolone resistance from a transferable plasmid in Shigella flexneri 2b. Antimicrob Agents Chemother 2005, 49(2):801-803.

35. Namboodiri SS, Opintan JA, Lijek RS, Newman MJ, Okeke IN: Quinolone resistance in Escherichia coli from Accra, Ghana. BMC Microbiol 2011, 11(1):44.
36. Okeke I: The microbial rebellion: trends and containment of antimicrobial resistance in Africa. In HIV/AIDS, illness, and African well-being. Volume 27. Edited by: Falola T, Heaton MM. Rochester, NY: University Of Rochester Press; 2007.

37. Aina BA, Tayo F, Taylor O: Cost implication of irrational prescribing of chloroquine in Lagos State general hospitals. I Infect Dev Ctries 2008, 2(1):68-72.

38. Aibinu I, Aednipekun E, Odugbemi T: Emergence of quinolone resistance amongst Escherichia coli strains isolated from clinical infections in some Lagos state hospitals, in Nigeria. Nig J HealthBiomed Sci 2004, 3(2):73-78.

39. Okeke I, Lamikanra A, Czeczulin J, Dubovsky F, Kaper J, Nataro J: Heterogeneous virulence of enteroaggregative Escherchia coli strains isolated from children in Southwest Nigeria. J Infect Dis 2000, 181:252-260.

40. Okeke IN, Fayinka ST, Lamikanra A: Antibiotic resistance in Escherichia coli from Nigerian students, 1986 1998. Emerg Infect Dis 2000, 6(4):393-396.

41. Lamikanra A, Ako-nai AK, Ogunniyi DA: Transferable antibiotic resistance in Escherichia coli isolated from healthy Nigerian school children. Int J Antimicrob Ag 1996, 7:59-64.

42. Djie-Maletz A, Reither K, Danour S, Anyidoho L, Saad E, Danikuu F, Ziniel P, Weitzel T, Wagner J, Bienzle U, et al: High rate of resistance to locally used antibiotics among enteric bacteria from children in Northern Ghana. J Antimicrob Chemother 2008, 61(6):1315-1318.

43. Le TM, Baker S, Le TP, Cao TT, Tran TT, Nguyen VM, Campbell Jl, Lam MY, Nguyen $T H$, Nguyen $W$, et al: High prevalence of plasmid-mediated quinolone resistance determinants in commensal members of the Enterobacteriaceae in Ho Chi Minh City, Vietnam. J Med Microbiol 2009, 58(Pt 12):1585-1592.

44. Escobar-Paramo P, Grenet K, Le Menac'h A, Rode L, Salgado E, Amorin C, Gouriou S, Picard B, Rahimy MC, Andremont A, et al: Large-scale population structure of human commensal Escherichia coli isolates. App/ Environ Microbiol 2004, 70(9):5698-5700.

45. Briales A, Rodriguez-Martinez JM, Velasco C, Diaz de Alba P, DominguezHerrera J, Pachon J, Pascual A: In vitro effect of qnrA1, qnrB1, and qnrS1 genes on fluoroquinolone activity against isogenic Escherichia coli isolates with mutations in gyrA and parC. Antimicrob Agents Chemother 2011, 55(3):1266-1269.

46. Aina BA, Tayo F, Taylor O, Eniojukan JF: Antimalarial prescribing patterns in state hospitals and selected parastatal hospitals in Lagos, Nigeria. Nig Q J Hosp Med 2009, 19(1):20-26.

47. Babalola O, Lamikanra A: Pattern of antibiotic purchases in community pharmacies in South Western Nigeria. J Soc Admin Pharm 2002, 19:33-38

48. Boni MF, Smith DL, Laxminarayan R: Benefits of using multiple first-line therapies against malaria. Proc Natl Acad Sci USA 2008, 105(37):14216-14221.

49. Bergstrom CT, Lo M, Lipsitch M: Ecological theory suggests that antimicrobial cycling will not reduce antimicrobial resistance in hospitals. Proc Natl Acad Sci USA 2004, 101(36):13285-13290.

50. Nkemngu N, Munong: Susceptibility patterns of Salmonella enterica serovar Typhi to 10 antibiotics in Cameroon. 6th International Conference on Typhoid Fever and Other Salmonelloses: 2005; Guilin, china 2005, 99.

51. Opintan JA, Newman MJ, Nsiah-Poodoh OA, Okeke IN: Vibrio cholerae 01 from Accra, Ghana carrying a class 2 integron and the SXT element. J Antimicrob Chemother 2008, 62(5):929-933.

\section{Pre-publication history}

The pre-publication history for this paper can be accessed here: http://www.biomedcentral.com/1471-2334/11/312/prepub

doi:10.1186/1471-2334-11-312

Cite this article as: Lamikanra et al:: Rapid evolution of fluoroquinoloneresistant Escherichia coli in Nigeria is temporally associated with fluoroquinolone use. BMC Infectious Diseases 2011 11:312. 